\title{
Cohomology with causally restricted supports
}

\author{
Igor Khavkine \\ Department of Mathematics, University of Trento \\ and TIFPA-INFN, Trento \\ Via Sommarive, 14, 38123 Povo (TN) Italy \\ igor.khavkine@unitn.it
}

April 16, 2018

\begin{abstract}
De Rham cohomology with spacelike compact and timelike compact supports has recently been noticed to be of importance for understanding the structure of classical and quantum Maxwell theory on curved spacetimes. Similarly causally restricted cohomologies of different differential complexes play a similar role in other gauge theories. We introduce a method for computing these causally restricted cohomologies in terms of cohomologies with either compact or unrestricted supports. The calculation exploits the fact that the de Rham-d'Alembert wave operator can be extended to a chain map that is homotopic to zero and that its causal Green function fits into a convenient exact sequence. As a first application, we use the method on the de Rham complex, then also on the Calabi (or Killing-Riemann-Bianchi) complex, which appears in linearized gravity on constant curvature backgrounds. We also discuss applications to other complexes, as well as generalized causal structures and functoriality.
\end{abstract}

Keywords: de Rham cohomology, Lorentzian manifold, causal structure, Calabi complex

MSC: $14 \mathrm{~F} 40,53 \mathrm{C} 50,58 \mathrm{~J} 10,58 \mathrm{~J} 45$

\section{Introduction}

Recently, a number of works on the structure of classical and quantum field theory on curved spacetimes $[15,47,6,19,5,31,34,36$ have made use of de Rham cohomology with spacelike compact supports. It appears in the characterizations of the center of Poisson (or quantum) algebra of observables of the Maxwell field and also of the degeneracy of the bilinear pairing between spacelike compactly supported solutions and compactly supported smearing functions (see Proposition 1 for a specific statement). Similar considerations appear in more general field theories [36, 34, though involving cohomologies of complexes that are different from the de Rham one. One example is the Calabi complex, which appears in linearized gravity on constant curvature backgrounds [36, Sec.4.4] 
(see Proposition 10 for a specific statement). Note that cohomologies with timelike compact supports as well as on-shell cohomologies (restricted to solution spaces of some particular hyperbolic differential operators) have also appeared in the same contexts. We shall loosely refer to all of these variations as causally restricted cohomologies or cohomologies with causally restricted supports.

It was noticed long ago [1] that non-trivial spacetime topology can influence in a non-trivial way the construction of the classical and quantum field theories. However, these effects had not been systematically investigated until recently. This may explain why neither the standard literature on differential geometry and topology, nor the literature on relativity seem to have considered 11 cohomologies with supports restricted by causal relations (like spacelike or timelike compactness). So, given their growing importance, they deserve independent investigation, which is the subject of this work. We introduce a method that allows us to compute the causally restricted cohomologies of a differential complex, provided that complex is equipped with extra structure similar to that found in Hodge theory [27, 33. The essentials of this method are illustrated on the case of the de Rham complex. Then, other applications and implications are discussed.

In Section 2, we briefly outline some well known geometric properties of the de Rham complex on a Lorentzian spacetime, as well as some basic facts of homological algebra. These properties form the core of our method and are reminiscent of the structure found in Hodge theory. Our method of computing causally restricted cohomologies is then illustrated in Section 3 and is used to express the various causally restricted de Rham cohomologies in terms of the standard de Rham cohomologies with unrestricted and compact supports. Section 4 applies the same method to the Calabi differential complex. The Calabi complex plays a role in linearized gravity on a constant curvature background analogous to that of the de Rham complex for Maxwell theory. Its structure is briefly introduced and shown analogous to that highlighted in Section 2, Then, in Section 4.4 its causally restricted cohomologies are computed in analogy with Section 3 Section 5 discusses a few related questions that have appeared in the study of gauge theories in the framework of locally covariant classical and quantum field theory. In particular, Sections 5.1 and [5.2 deal with the behavior of the causally restricted cohomology groups under changes of causal structure and under embeddings, and Section 5.3 briefly describes how the methods applied to the de Rham and Calabi examples could be generalized to other differential complexes that arise in the study of general field theories with constrains and gauge invariance 36, 34. Finally, Section 6 concludes with a discussion of our results.

It should be mentioned that results very similar to those in Section 3 have been obtained independently in the recent work [5], though by a different methods. Those methods are very specific to the de Rham complex, including its invariance properties under topological homotopies. Such strong invariance properties certainly do not hold for other differential complexes. So it is noteworthy that the content of our Sections 4 and 5 goes beyond [5] in several directions.

\footnotetext{
${ }^{1}$ A notable exception is [39], which, as a byproduct of a different investigation, computed a few low degree cohomology groups with spacelike compact supports or restricted to solutions of the wave equation, but only on Minkowski space.
} 


\section{Preliminaries}

Fix an $n$-dimensional smooth manifold $M(n \geq 2)$ with a Lorentzian metric $g$ such that $(M, g)$ is an oriented, time-oriented space, globally hyperbolic spacetime [52, 32, 43, 4. Recall that, according to the Geroch splitting theorem, there exists a diffeomorphism $M \cong \mathbb{R} \times \Sigma$ (non-unique, of course) where the corresponding projection $t: M \rightarrow \mathbb{R}$ is a Cauchy temporal function [25, 9, 8].

Let $\Omega^{p}(M)$ denote the linear space of differential $p$-forms on $M$ and let $\mathrm{d}: \Omega^{p}(M) \rightarrow \Omega^{p+1}(M)$ denote the de Rham differential, which together form the de Rham complex

$$
0 \longrightarrow \Omega^{0}(M) \stackrel{\mathrm{d}}{\longrightarrow} \Omega^{1}(M) \stackrel{\mathrm{d}}{\longrightarrow} \cdots \stackrel{\mathrm{d}}{\longrightarrow} \Omega^{n}(M) \longrightarrow 0,
$$

This sequence of maps being a complex means that each pair of successive maps compose to zero, $\mathrm{d} \circ \mathrm{d}=0$. Its cohomology in degree $p$ is defined and denoted by

$$
H^{p}(M):=\frac{\operatorname{ker}\left(\mathrm{d}: \Omega^{p}(M) \rightarrow \Omega^{p+1}(M)\right)}{\operatorname{im}\left(\mathrm{d}: \Omega^{p-1}(M) \rightarrow \Omega^{p}(M)\right)} .
$$

The cohomology of any other complex is defined in a similar way. It is well known that this de Rham cohomology is isomorphic, $H^{p}(M) \cong H^{p}(M, \mathbb{R})$, to the singular cohomology of $M$ with coefficients in $\mathbb{R}$ [11, Thm.15.8], to the Cech cohomology of $M$ with coefficients in $\mathbb{R}$ [11, Thm.8.9], and to the sheaf cohomology of $M$ with coefficients in the sheaf of locally constant $\mathbb{R}$-valued functions $[11$, Prp.10.6], all of which being isomorphic are denoted by $H^{p}(M, \mathbb{R})$. If we replace $\Omega^{p}(M)$ in (11) with $\Omega_{c}^{p}(M)$, the linear space of differential $p$-forms with compact support, the corresponding de Rham cohomology of $M$ with compact supports, which satisfies the following isomorphism: $H_{c}^{p}(M)^{*} \cong H^{p}(M, \mathbb{R})$. That isomorphism is implemented by a non-degenerate bilinear pairing between $\Omega^{p}(M)$ and $\Omega_{c}^{n-p}(M)$,

$$
\langle\alpha, \beta\rangle=\int_{M} \alpha \wedge \beta
$$

which descends to a non-degenerate bilinear pairing between $H^{p}(M)$ and $H_{c}^{p}(M)$. This result is known as Poincaré duality [11, Rmk.5.7].

Using the Hodge star operator $*: \Omega^{p}(M) \rightarrow \Omega^{n-p}(M)$ associated to the metric $g$, we can define the de Rham co-differential $\delta=* \mathrm{~d} *: \Omega^{p}(M) \rightarrow \Omega^{p-1}(M)$. Next, we define the so-called de Rham-d'Alembertian or wave operator $\square: \Omega^{p}(M) \rightarrow$ $\Omega^{p}(M)$,

$$
\square=\mathrm{d} \delta+\delta \mathrm{d}
$$

This operator differs from the simple tensor d'Alembertian $\nabla_{a} \nabla^{a}$ by terms of lower differential order. From its very definition, we see that the d'Alembertian is a cochain map from the de Rham complex to itself, $\mathrm{d} \square=\square$ d, which is moreover cochain homotopic to zero, with the co-differential $\delta$ the corresponding cochain homotopy. That is, it induces the zero map from $H^{p}(M)$ to itself. The following diagram illustrates the discussion:

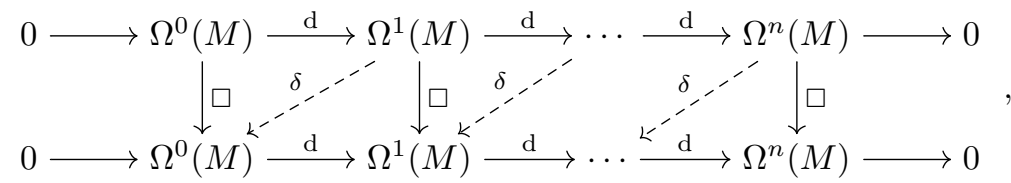


where the rows constitute (de Rham) complexes, the solid arrows commute, and the dashed arrows illustrate the cochain homotopy. This is an important observation that will be used in an essential way in Section 3 Note that the formula (31) is analogous to the formula for the Hodge-de Rham Laplacian in Riemannian geometry. There, the observation that this Laplacian is homotopic to zero lies at the foundation of Hodge theory [27, 33.

The causal structure on $M$ defined by the Lorentzian metric $g$ allows us to restrict the supports of differential forms in other ways as well. Recall that, for a subset $S \subseteq M$, by $J^{ \pm}(S)$ we denote the subset of $M$ that can be reached from $S$ by piecewise smooth, future (+) or past (-) directed causal curves, while $J(S)=J^{+}(S) \cup J^{-}(S)$. A closed set $S \subseteq M$ is said to be retarded if $S \subseteq J^{+}(K)$ for some compact $K$, advanced if $S \subseteq J^{-}(K)$ for some compact $K$, spacelike compact if it $S \subseteq J(K)$ for some compact $K$, past compact if $S \cap J^{-}(K)$ is compact for every compact $K$, future compact if $S \cap J^{+}(K)$ is compact for every compact $K$, and timelike compact if $S$ is both past and future compact [46, 2]. Timelike compactness is also equivalent to the property of having compact intersection with every spacelike compact set. Let $\Omega_{X}^{p}(M)$, with $X=+,-, s c, p c, f c$ or $t c$, denote the linear space of differential $p$-forms with, respectively, retarded, advanced, spacelike compact, past compact, future compact or timelike compact supports. For brevity, we refer to these spaces as space of forms with causally restricted supports.

Of course, since differential operators preserve supports, $\square$ also restricts to $\square: \Omega_{c}^{p}(M) \rightarrow \Omega_{c}^{p}(M)$. By the same reasoning, the spaces of forms with causally restricted supports are also preserved by both d and $\square$. We define de Rham cohomology with causally restricted supports in the obvious way and denote it by $H_{X}^{p}(M)$, with $X=+,-, s c, p c, f c$ or $t c$. Let $\Omega_{\square}^{p}(M)$ and $\Omega_{\square, X}^{p}(M)$ denote the kernel of the wave operator $\square$, also known as its solution space, in the spaces of forms with corresponding supports. Finally, by the cochain map property, the de Rham differential restricts to the kernel of the wave operator, hence defining the de Rham cohomology groups $H_{\square}^{p}(M)$ and $H_{\square, X}^{p}(M)$ of solutions.

The specific way in which these causally restricted cohomologies are of importance in Maxwell gauge theory is summarized in the following proposition. For definiteness of notation let us fix a $\chi \in C^{\infty}(M)$ that is 1 in the future of a Cauchy surface $\Sigma_{+}$and 0 in the past of another Cauchy surface $\Sigma_{-}$. The following is a special case of the general result [36, Thm.3.2].

Proposition 1. Maxwell gauge theory [36, Sec.4.2] induces a symplectic form on $\Omega_{\square, s c}^{1}(M)$ [36, Def.3.10] that is non-degenerate when (a) the bilinear form on $H_{s c}^{1}(M) \times H_{c}^{n-1}(M)$ induced by $\langle\alpha, \beta\rangle=\int_{M} \alpha \wedge \beta$ is non-degenerate and (b) the bilinear form on $H_{\square, s c}^{1}(M)$ induced by $\langle\alpha, \beta\rangle_{\square}=\int_{M} \alpha \wedge * \square(\chi \beta)$ is nondegenerate (where $*$ denotes the Hodge dual).

From the proof of that proposition it also follows that degeneracies in (a) and (b) can imply degeneracies in the corresponding (pre-)symplectic structure.

The wave operator on a globally hyperbolic Lorentzian manifold is well known to be Green hyperbolic. That is, it has advanced and retarded Green functions denoted respectively $\mathrm{G}_{+}$and $\mathrm{G}_{-}, \mathrm{G}_{ \pm}: \Omega_{c}^{p}(M) \rightarrow \Omega_{+}^{p}(M)$. Since $\square$ commutes with $\mathrm{d}$, then so do $\mathrm{G}_{+}$and $\mathrm{G}_{-}$. The form $\beta=\mathrm{G}_{ \pm}[\alpha]$ is the unique solution of $\square \beta=\alpha$ with, respectively, retarded or advanced support. The domain of definition of the Green functions can be extended, in a unique way, to 
$\Omega_{X}^{p}(M)$ for $X=+,-, p c$ or $f c$. Then, the maps

$$
\square: \Omega_{Y}^{p}(M) \rightarrow \Omega_{Y}^{p}(M), \quad \mathrm{G}_{X}: \Omega_{Y}^{p}(M) \rightarrow \Omega_{Y}^{p}(M)
$$

are mutually inverse bijections, whenever $X=+$ and $Y=+$ or $p c$, or $X=-$ and $Y=-$ or $f c$. The combination $\mathrm{G}=\mathrm{G}_{+}-\mathrm{G}_{-}$is known as the causal Green function and fits into the following, in our terminology Green-hyperbolic, exact sequences [3, 26, 36, 34, 2]

$$
\begin{aligned}
& 0 \longrightarrow \Omega_{c}^{p}(M) \stackrel{\square}{\longrightarrow} \Omega_{c}^{p}(M) \stackrel{\mathrm{G}}{\longrightarrow} \Omega_{s c}^{p}(M) \stackrel{\square}{\longrightarrow} \Omega_{s c}^{p}(M) \longrightarrow 0, \\
& 0 \longrightarrow \Omega_{t c}^{p}(M) \stackrel{\square}{\longrightarrow} \Omega_{t c}^{p}(M) \stackrel{\mathrm{G}}{\longrightarrow} \Omega^{p}(M) \stackrel{\square}{\longrightarrow} \Omega^{p}(M) \longrightarrow 0 .
\end{aligned}
$$

Note that, according to the above formulas, we can represent the space of solutions with spacelike compact or unrestricted support either as

$$
\begin{gathered}
\Omega_{\square, X}^{p}(M)=\operatorname{ker} \square \subset \Omega_{X}^{p}(M) \\
\text { or } \quad \Omega_{\square, X}^{p}(M)=\mathrm{G}\left[\Omega_{Y}^{p}(M)\right]=\Omega_{Y}^{p}(M) / \square \Omega_{Y}^{p}(M),
\end{gathered}
$$

with $X=s c$ and $Y=c$, or $X$ empty and $Y=t c$, respectively. On the other hand, we have trivial solution spaces $\Omega_{\square, X}^{p}(M)=\{0\}$ when $X=+,-, p c$ or $f c$.

The existence of the Green-hyperbolic exact sequences will allow us to later make use of the following elementary result of homological algebra [11, p.17]. Let $A^{\bullet}=\left(A^{p}, \mathrm{~d}\right)$ be a cochain complex, and similarly for $B^{\bullet}$ and $C^{\bullet}$. It is well known that a short exact sequence of cochain maps (maps commuting with the differentials d),

$$
0 \longrightarrow A^{\bullet} \stackrel{f}{\longrightarrow} B^{\bullet} \stackrel{g}{\longrightarrow} C^{\bullet} \longrightarrow 0,
$$

induces a long exact sequence in cohomology,

$$
\begin{array}{r}
\left.0 \longrightarrow H^{0}\left(A^{\bullet}, \mathrm{d}\right) \stackrel{[f]}{\longrightarrow} H^{0}\left(B^{\bullet}, \mathrm{d}\right) \stackrel{[g]}{\longrightarrow} H^{0}\left(C^{\bullet}, \mathrm{d}\right)\right] \\
\longrightarrow H^{1}\left(A^{\bullet}, \mathrm{d}\right) \stackrel{[f]}{\longrightarrow} H^{1}\left(B^{\bullet}, \mathrm{d}\right) \stackrel{[g]}{\longrightarrow} H^{1}\left(C^{\bullet}, \mathrm{d}\right) \stackrel{[\mathrm{d}]}{\longrightarrow} \cdots
\end{array}
$$

The maps $[f],[g]$ are induced by the corresponding cochain maps, while the $[\mathrm{d}]$ maps are induced by the differentials of the complexes (hence our notation for them) and are known as connecting homomorphisms.

\section{Computation of cohomology groups}

In this section, we state and prove our main results on de Rham cohomology with causally restricted supports. We rely essentially on the properties of the wave operator and its Green functions, as summarized in Section 2, The important properties are that the wave operator $\square$ is cochain homotopic to zero, and the way its range and kernel are characterized using the causal Green function G. In particular, we do not explicitly rely on the invariance properties of the de Rham complex under topological homotopies. 
Theorem 2. De Rham cohomology $H_{X}^{p}(M)$, with $X=+,-, p c$ or $f c$, is trivial.

Proof. Let $X=+,-, p c$ or $f c$. Then, as was noted in Section 2, the wave operator is a cochain map of the corresponding de Rham complex into itself, is invertible [Equation (5)] and cochain homotopic to zero [Equation (3)]. Thus, it induces a map in cohomology that is both invertible and equal to zero, which can only mean that all the cohomologies are trivial. More concretely, given any closed $\alpha \in \Omega_{X}^{p}(M)$, the identity $\mathrm{d}\left(\delta \mathrm{G}_{X}[\alpha]\right)=\mathrm{G}_{X}[(\mathrm{~d} \delta+\delta \mathrm{d}) \alpha]=\alpha$ shows that it is also exact.

Theorem 3. We have the isomorphisms

$$
\begin{aligned}
H_{s c}^{p}(M) & \cong H_{c}^{p+1}(M), & H_{\square, s c}^{p} & \cong H_{c}^{p}(M) \oplus H_{c}^{p+1}(M), \\
H_{t c}^{p}(M) & \cong H^{p-1}(M), & \text { and } H_{\square}^{p}(M) & \cong H^{p}(M) \oplus H^{p-1}(M),
\end{aligned}
$$

with the convention that all cohomologies vanish in degree $p$ for $p<0$ or $p>n$.

Proof. Recall again from Section 2 that both the wave operator $\square$ and its causal Green function $\mathrm{G}$ commute with $\mathrm{d}$ and hence constitute cochain maps between the de Rham complexes with appropriate supports, inducing maps in cohomology. Moreover, since $\square$ is cochain homotopic to zero [Equation (3)] , it induces the zero map in cohomology.

Let us start with spacelike compact supports. We can break the exact sequence in (6) into two short exact sequences of complexes:

$$
\begin{gathered}
0 \longrightarrow \Omega_{c}^{p}(M) \stackrel{\square}{\longrightarrow} \Omega_{c}^{p}(M) \stackrel{\mathrm{G}}{\longrightarrow} \Omega_{\square, s c}^{p}(M) \longrightarrow 0, \\
0 \longrightarrow \Omega_{\square, s c}^{p}(M) \stackrel{\subset}{\longrightarrow} \Omega_{s c}^{p}(M) \stackrel{\square}{\longrightarrow} \Omega_{s c}^{p}(M) \longrightarrow 0 .
\end{gathered}
$$

Because $\square$ always induces the zero map, $[\square]=0$, the corresponding long exact sequences in cohomology [cf. Equation (11)] break up into the following short exact sequences:

$$
\begin{gathered}
0 \longrightarrow H_{c}^{p}(M) \stackrel{[\mathrm{G}]}{\longrightarrow} H_{\square, s c}^{p}(M) \stackrel{[\mathrm{d}]}{\longrightarrow} H_{c}^{p+1}(M) \longrightarrow 0, \\
0 \longrightarrow H_{s c}^{p-1}(M) \stackrel{[\mathrm{d}]}{\longrightarrow} H_{\square, s c}^{p}(M) \stackrel{[\subset]}{\longrightarrow} H_{s c}^{p}(M) \longrightarrow 0,
\end{gathered}
$$

again with the convention that any $H_{X}^{p}(M)$ vanishes for $p<0$ or $p>n$. Since we are dealing with real vector spaces, any exact sequence splits, giving us the isomorphisms

$$
H_{c}^{p}(M) \oplus H_{c}^{p+1}(M) \cong H_{\square, s c}^{p}(M) \cong H_{s c}^{p-1}(M) \oplus H_{s c}^{p}(M) .
$$

Given that $H_{c}^{0}(M)$ and $H_{s c}^{-1}(M)$ both vanish $(M$ is non-compact and there are no forms in degree $p=-1$ ), plugging $p=0$ into the above isomorphism implies $H_{s c}^{0}(M) \cong H_{c}^{1}(M)$. Proceeding by induction on $p$, we can check that $H_{s c}^{p}(M) \cong H_{c}^{p+1}(M)$ for all $p$. Thus, we obtain the isomorophisms

$$
\begin{aligned}
H_{s c}^{p}(M) & \cong H_{c}^{p+1}(M), \\
H_{\square, s c}^{p}(M) & \cong H_{c}^{p}(M) \oplus H_{c}^{p+1}(M) .
\end{aligned}
$$


Applying the same argument to the exact sequence (7), we obtain the isomorphisms

$$
\begin{aligned}
H_{t c}^{p}(M) & \cong H^{p-1}(M), \\
H_{\square}^{p}(M) & \cong H^{p}(M) \oplus H^{p-1}(M) .
\end{aligned}
$$

This completes the proof.

Let $\Sigma \subset M$ be a Cauchy surface. Recall that, by the smooth Geroch splitting theorem, we can always smoothly factor $M \cong \mathbb{R} \times \Sigma$. This observation results in

Corollary 4. We have the isomorphisms

$$
\begin{aligned}
H_{s c}^{p}(M) & \cong H_{c}^{p}(\Sigma), & H_{\square, s c}^{p}(M) & \cong H_{c}^{p}(\Sigma) \oplus H_{c}^{p-1}(\Sigma) \\
H_{t c}^{p}(M) & \cong H^{p-1}(\Sigma), & \text { and } H_{\square}^{p}(M) & \cong H^{p}(\Sigma) \oplus H^{p-1}(\Sigma),
\end{aligned}
$$

with the convention that all cohomologies vanish in degree $p$ for $p<0$ or $p>n$.

Proof. The splitting $M \cong \mathbb{R} \times \Sigma$ shows that $M$ is homotopic to $\Sigma$. Hence, by the homotopy invariance of de Rham cohomologies with unrestricted supports, we have the isomorphism $H^{p}(M) \cong H^{p}(\Sigma)$. On the other hand, Poincare duality induces the isomorphism $H_{c}^{p}(M) \cong H_{c}^{p-1}(\Sigma)$. Therefore, the desired conclusion follows directly from these identities in combination with Theorem 3 .

Finally, knowing the respective de Rham cohomologies with spacelike and timelike compact supports, we have the following generalization of the Poincaré lemma.

Corollary 5. The non-degenerate bilinear pairing between $\Omega_{s c}^{p}(M)$ and $\Omega_{t c}^{n-p}(M)$ descends to a non-degenerate bilinear pairing between $H_{s c}^{p}(M)$ and $H_{t c}^{n-p}(M)$. There exists also a non-degenerate bilinear pairing between $H_{\square, s c}^{p}(M)$ and $H_{\square}^{n-p}(M)$.

Proof. A consequence of Theorem 3 is that $H_{s c}^{p}(M) \cong H_{c}^{p+1}(M)$ and $H_{t c}^{n-p}(M)=$ $H^{n-p-1}(M)$. So, the usual Poincaré duality establishes that $H_{s c}^{p}(M)^{*} \cong H_{t c}^{n-p}(M)$. The isomorphism can be exhibited by bilinear pairing, which descends from the standard bilinear pairing between $\Omega_{s c}^{p}(M)$ and $\Omega_{t c}^{n-p}(M)$, tracing its effect throughout the proof of Theorem 3 Its non-degeneracy is also a consequence of the Poincaré lemma applied to $H_{c}^{p}(M)$ and $H^{n-p}(M)$.

It also follows from Theorem 3 that $H_{\square, s c}^{p}(M) \cong H_{c}^{p}(M) \oplus H_{c}^{p+1}(M)$ and $H_{\square}^{n-p}(M) \cong H^{n-p}(M) \oplus H^{n-p-1}(M)$. Again, the usual Poincaré duality establishes the isomorphism $H_{\square, s c}^{p}(M)^{*} \cong H_{\square}^{n-p}(M)$. The isomorphism can be exhibited by a bilinear pairing between $\Omega_{\square, s c}^{p}(M)$ and $\Omega_{\square}^{n-p}(M) \cong \Omega_{t c}^{n-p}(M) / \square \Omega_{t c}^{n-p}(M)$, defined by the latter identity and the self-adjointness of $\square$ with respect to our pairing between forms. Again, tracing this pairing through the proof of Theorem 3 and appealing to the standard Poincaré duality establishes its nondegeneracy.

As already discussed in the introduction, the importance of knowing the above cohomology groups is important for understanding the (pre)symplectic and Poisson structure of classical field theories, as emphasized in [36, 34, 47, 6, 5]. The same result as Corollary 4 was obtained independently in [5]. As a matter of fact, the method of $[5$ can be seen as a special case of our homological calculation, as discussed more explicitly at the end of Section 5.1.2. 


\section{Calabi or Killing-Riemann-Bianchi complex}

In 36, 34, it was pointed out that the construction of the symplectic and Poisson structures on the phase space of field theories with constraints and/or gauge invariance can be done using a general framework, provided a given field theory satisfies certain geometric conditions. These conditions include the existence of certain differential complexes that extend the operators that constitute the constraints and that generate the gauge transformations. For Maxwell (and similar) theories, all of these complexes are invariably part of the de Rham complex 36 . Secs.4.2-3]. On the other hand, for linearized gravity, one has to use something different. Unfortunately, the explicit form of these differential complexes is not currently known for linearized gravity on an arbitrary background 36. Sec.4.4]. However, in the special case of constant curvature backgrounds, the answer is known and it is the so-called Calabi complex [13. It is likely that, once an explicit understanding of the corresponding differential complexes for more general backgrounds is achieved, the general framework of [36, 34] would supersede recent covariant treatments of the quantization of linearized gravity like [18, 30.

The Calabi complex provides a fine resolution [12, Sec.II.9] of the sheaf of Killing vectors, similarly to how the de Rham complex provides a fine resolution of the sheaf of locally constant functions. The cohomology of a sheaf (a rather abstract object) is isomorphic to the cohomology of the complex of global sections of a fine resolution of the same sheaf (a more concrete object), which is what makes fine resolutions significant [12, Thm.II.4.1]. As such, the Calabi complex has been studied in some literature on the deformation of constant curvature geometric structures [13, 7, 29, 23, 24, 44, 16. Because its structure is substantially different from the de Rham complex, we summarize some of its relevant properties in Sections 4.1 through 4.3 before concentrating on its causally restricted cohomologies in Section 4.4. Many of these properties are scattered throughout or are simply not available in the existing literature. We defer a fuller discussion of the Calabi complex, which collects these properties and their proofs, to 35. However, all that we really need for the purposes of Section 4.4 is the existence of differential operators listed in Section 4.2 and the identities between them. Since these differential operators are explicitly given, the identities can in principle be verified by direct calculation.

\subsection{Tensor bundles}

We will present later a differential complex whose nodes are sections of tensor bundles that are not so easy to express in conventional notation. So, let us introduce the following short-hands. We denote the cotangent bundle by $V M=$ $T^{*} M$ and the bundle of metrics (symmetric, covariant 2-tensors) by $S^{2} M=$ $S^{2} T^{*} M$. Let $R M \subset\left(T^{*}\right)^{4} M$ denote the sub-bundle of covariant 4-tensors that satisfy the algebraic symmetries of the Riemann tensor $\left(R_{(a b) c d}=R_{a b(c d)}=\right.$ $\left.R_{a b c d}-R_{c d a b}=R_{[a b c] d}=0\right)$. Next, we let $B M \subset\left(T^{*}\right)^{5} M$ denote the target bundle of the Bianchi operator $\nabla_{[a} R_{b c]}$. At this point it is convenient to notice that the fiber of each of these bundles carries 20 an irreducible representation of $\operatorname{GL}(n)$, with $n=\operatorname{dim} M$. In fact, it is easiest to describe the remaining tensor bundles in terms of the irreducible $\mathrm{GL}(n)$ representation carried by their fibers. So let $C_{l} M \subset\left(T^{*}\right)^{l+2} M$ (with $C$ standing for Calabi) denote the sub-bundles 
of covariant $(l+2)$-tensors with the corresponding irreducible representations listed in Table 1, which also lists their fiber ranks. It is consistent for us to assign $C_{0} M \cong V M, C_{1} M \cong S^{2} M$ and $C_{2} M \cong R M$ and $C_{3} M \cong B M$. Recall that, on an $n$-dimensional manifold, the largest rank of a fully antisymmetric tensor is $n$. So the bundles $C_{l} M$ become trivial (zero fiber rank) for $l>n$.

Given two $S^{2} M$ tensors, we can construct an $R M$ tensor out of them using the formula

$$
(g \odot h)_{a b c d}=g_{a c} h_{b d}-g_{b c} h_{a d}-g_{a d} h_{b c}+g_{b d} h_{a c} .
$$

In fact, the above formula represents a $\operatorname{GL}(n)$-equivariant map between $S^{2} \otimes S^{2}$ and $R$ (where we use the bundle prefixes to stand in for the corresponding irreducible representations). The decomposition of the $S^{2} \otimes S^{2}$ tensor product has only one copy of $R$, so by Schur's lemma such a map is unique, up to an overall rescaling. The same argument can be repeated for the tensor product $S^{2} \otimes Y$, where $Y$ corresponds to any other Young diagram. This tensor product decomposes into irreducible subrepresentations without multiplicities. Then the projection onto any of the subrepresentations $Y^{\prime}$ is well defined up to a rescaling. If we fix sections $g$ of $S^{2} M$ and $h$ of $Y M$, these projections define a bilinear operation between $g$ and $h$ with the result a section of $Y^{\prime} M$. We use the following explicit formulas:

$$
\begin{aligned}
(g \odot t)_{a b c: d e}= & +g_{a d} t_{b c: e}+g_{b d} t_{c a: e}+g_{c d} t_{a b: e} \\
& -g_{a e} t_{b c: d}-g_{b e} t_{c a: d}-g_{c e} t_{a b: d} \\
(g \odot t)_{a b c d: e f}= & +g_{a e} t_{b c d: f}-g_{b e} t_{c d a: f}+g_{c e} t_{d a b: f}-g_{d e} t_{a b c: f} \\
& -g_{a f} t_{b c d: e}+g_{b f} t_{c d a: e}-g_{c f} t_{d a b: e}+g_{d f} t_{a b c: e}
\end{aligned}
$$

Note that a tensor with indices written as in $t_{a b c: d e}$ has the symmetry type $(2,2,1)$, while $t_{a b c: d}$ corresponds to the symmetry type $(2,1,1)$, and so on. The colon : is used purely as a visuall aid to separate groups of indices belonging to different columsn of a Young diagram.

The metric $g_{a b}$ itself, an $S^{2} M$ tensor, can now be used to produce an $R M$ tensor,

$$
(g \odot g)_{a b: c d}=2\left(g_{a c} g_{b d}-g_{b c} g_{a d}\right),
$$

which is obviously covariantly constant. In fact, a constant curvature spacetime must have (covariant) Riemann tensor, Ricci tensor and Ricci scalar of the following form

$$
\bar{R}_{a b c d}=\frac{k}{n(n-1)}\left(g_{a c} g_{b d}-g_{b c} g_{a d}\right), \quad \bar{R}_{a c}=\frac{k}{n} g_{a c}, \quad \bar{R}=k .
$$

We have decorated these quantities with a bar to indicate the fact that we shall fix a constant curvature background metric $g$ and consider perturbations on it. For our purposes, we also require that the Lorentzian manifold $(M, g)$ is globally hyperbolic.

We should note that solutions of Einstein equations (including a possible cosmological constant term) with constant curvature includes Minkowski space $(k=0)$, de Sitter space $(k>0)$ and anti-de Sitter space $(k<0)$. There is (up to isometry) a unique simply connected version of each of these cases [32, §5.1-2]. Other examples may be obtained by taking quotients thereof with respect to a 
Table 1: It is conventional to label irreducible GL $(n)$ representations by Young diagrams [21]. Recall that a Young diagram with $k$ cells of type $\left(r_{1}, r_{2}, \ldots\right)$ consists of a number of rows of non-increasing lengths $r_{i}, r_{i+1} \leq r_{i}$, such that $\sum_{i} r_{i}=k$. Given a Young diagram with $k$ cells, an instance of the corresponding irreducible $\mathrm{GL}(n)$ representation class can be realized as the image of the space of covariant $k$-tensors after two projections: assign an independent tensor index to each cell of the diagram, symmetrize over each row, antisymmetrize over each column.

The table below lists the tensor bundles of the Calabi complex, the corresponding irreducible GL $(n)$ representations (labeled by Young diagrams), and their fiber ranks, for $\operatorname{dim} M=n$. The rank is given by the famous hook formula, which is the following fraction. The numerator is the product of the following numbers: place $n$ in the top left cell, increase by 1 to the right and decrease by 1 down, until all cells are filled. The denominator is the product of the following numbers: fill a given cell with the number of cells constituting a hook with vertex at the given location, extending to the right and down [21].

\begin{tabular}{|c|c|c|}
\hline bundle & Young diagram & fiber rank \\
\hline$V M \cong C_{0} M$ & & $n$ \\
\hline$S^{2} M \cong C_{1} M$ & & $\frac{n(n+1)}{2}$ \\
\hline$R M \cong C_{2} M$ & & $\frac{n^{2}\left(n^{2}-1\right)}{12}$ \\
\hline$B M \cong C_{3} M$ & & $\frac{n^{2}\left(n^{2}-1\right)(n-2)}{24}$ \\
\hline \multirow{3}{*}{$C_{l} M$} & 1 & \multirow{3}{*}{$\frac{n^{2}\left(n^{2}-1\right)(n-2) \cdots(n-l+1)}{2(l+1) l(l-2) !}$} \\
\hline & 2 & \\
\hline & $\begin{array}{l}: \\
l\end{array}$ & \\
\hline
\end{tabular}


discrete subgroup, thus changing the topology. The list of possibilities is thus exhausted by considering open subsets of such quotients. Some examples will not be globally hyperbolic (like anti-de Sitter space or quotients of Minkowski space with respect to timelike translations) and thus excluded from part of our discussion.

\subsection{Differential operators}

Now, we introduce a number of differential operators between the tensor bundles that we have defined. For convenience of notation, we denote the space of sections of a bundle by the same symbol as the bundle itself. These operators fit into the following diagram:

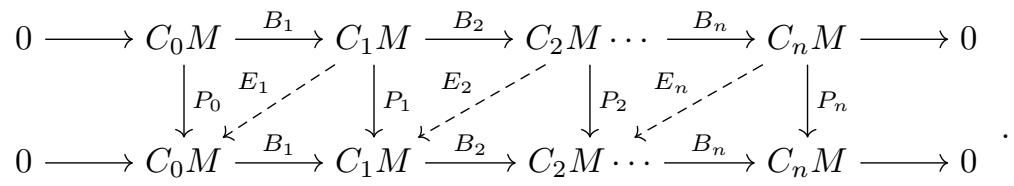

All the solid arrows commute and the rows constitute (cochain) complexes. The vertical maps are then necessarily cochain maps. They happen to satisfy the identities $P_{l}=E_{l+1} \circ B_{l+1}+B_{l} \circ E_{l}$, which means that they are null-homotopic, with the $E_{l}$ supplying the corresponding cochain homotopy.

Below, we give explicit formulas for all these differential operators in dimension $n=4$. More details can be found in 35, which draws from the earlier works $13,7,29,23,24,44,16$. As we shall see, for low indices they are well known in the relativity literature. However, the relations between them in terms of fitting into the above diagram do not seem to have been fully noted.

The Calabi differential complex is given by

$$
\begin{aligned}
B_{1}[v]_{a b}= & \nabla_{a} v_{b}+\nabla_{b} v_{a}, \\
B_{2}[h]_{a b: c d}= & \left(\nabla_{(a} \nabla_{c)} h_{b d}-\nabla_{(b} \nabla_{c)} h_{a d}-\nabla_{(a} \nabla_{d)} h_{b c}+\nabla_{(b} \nabla_{d)} h_{a c}\right) \\
& +k \frac{1}{n(n-1)}(g \odot h)_{a b: c d}, \\
B_{3}[r]_{a b c: d e}= & 3 \nabla_{[a} r_{b c]: d e}=\nabla_{a} r_{b c: d e}+\nabla_{b} r_{c a: d e}+\nabla_{c} r_{a b: d e}, \\
B_{4}[b]_{a b c d: e f}= & 4 \nabla_{[a} b_{b c d]: e f} \\
= & \nabla_{a} b_{b c d: e f}-\nabla_{b} b_{c d a: e f}-\nabla_{c} b_{d a b: e f}-\nabla_{d} b_{a b c: e f}, \\
B_{l}[b]_{a_{1} \cdots a_{l}: b c}= & l \nabla_{\left[a_{1}\right.} b_{\left.a_{2} \cdots a_{l}\right]: b c} \quad(l \geq 3),
\end{aligned}
$$

where $\left(a_{1} \cdots a_{l}\right)$ and $\left[a_{1} \cdots a_{l}\right]$ denote respectively complete idempotent symmetrization and antisymmetrization of a group of indices [52, Eq.2.4.3-4]. Recall also that the colon : is used purely as a visual aid to separate groups of indices belonging to different columns of the Young diagrams in Table 1 The details showing that these operators have the desired symmetry properties and indeed define a complex, $B_{l+1} \circ B_{l}=0$, which is moreover elliptic 2 can be found in 35 .

\footnotetext{
${ }^{2} \mathrm{~A}$ complex of differential operators is elliptic if the corresponding complex of symbol maps is exact for every non-zero covector.
} 
It is interesting to note the following relations with well known differential operators in relativity. The Killing operator is $K[h]=B_{1}[h]$. The linearized Riemann tensor is $\dot{R}[h]=-\frac{1}{2} B_{2}[h]+k \frac{2}{n(n-1)}(g \odot h)$, where the all covariant non-linear Riemann tensor is expanded as $R[g+\lambda h]_{a b: c d}=\bar{R}_{a b: c d}+\lambda \dot{R}[h]_{a b: c d}$ (convention of [52]). The background Bianchi operator is $\bar{B}[r]=B_{3}[r]$, with $\bar{B}[\bar{R}]=0$. Finally, though the name is not standard, it is meaningful to call $B_{4}[b]$ a higher Bianchi operator. Thus, it would also make sense to refer to the Calabi complex as the Killing-Riemann-Bianchi complex. This complex also happens to be locally exact 13 13, 35. Thus, according to the general machinery of sheaf theory, the Calabi complex provides a fine resolution of the sheaf of Killing vectors (or Killing sheaf) $\mathcal{K}_{g}$ on the Lorentzian manifold $(M, g)$ [35, Sec.3]. This observation immediately gains us the following

Proposition 6 (Calabi [13). The (unrestricted) cohomology $H C^{l}(M, g)=$ ker $B_{l+1} / \mathrm{im} B_{l}$ of the Calabi complex is isomorphic to the sheaf cohomology $H^{\bullet}\left(M, \mathcal{K}_{g}\right)$ of the sheaf of Killing vectors on any spacetime $(M, g)$ of constant curvature.

Calabi's proof was rather elementary and relied on the specific structure of this complex. Unfortunately, his method does not generalize easily to other differential complexes. So, we discuss below a different method to get local exactness, which relies mostly on the ellipticity of the Calabi complex, a property which is expected to be shared by other complexes of interest.

Next, we give explicitly the homotopy differential operators

$$
\begin{aligned}
& E_{1}[h]_{a}=D[h]_{a}=\nabla^{b} h_{a b}-\frac{1}{2} \nabla_{a} h, \\
& E_{2}[r]_{a: b}=\operatorname{tr}[r]_{a: b}=r_{a c: b}{ }^{c}, \\
& E_{3}[b]_{a b: c d}=\nabla^{e} b_{e a b: c d}+\frac{1}{2} \nabla^{e}\left(b_{c a b: d e}-b_{d a b: c e}\right) \\
& -\frac{1}{2}\left(\nabla_{c} b_{a b e: d}{ }^{e}-\nabla_{d} b_{a b e: c^{e}}\right) \\
& -\frac{1}{2}\left(\nabla_{a} b_{c b e: d}{ }^{e}-\nabla_{a} b_{d b e: c} c^{e}\right. \\
& \left.+\nabla_{b} b_{a c e: d}{ }^{e}-\nabla_{b} b_{a d e: c^{e}}\right), \\
& E_{4}[b]_{a b c: d e}=\nabla^{f} b_{f a b c: d e}+\frac{1}{3} \nabla^{f}\left(b_{d a b c: e f}-b_{e a b c: d f}\right) \\
& +\frac{1}{3}\left(\nabla_{d} b_{a b c f: e^{f}}-\nabla_{e} b_{a b c f: d^{f}}\right)
\end{aligned}
$$

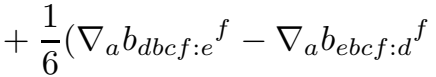

$$
\begin{aligned}
& +\nabla_{b} b_{a d c f: e^{f}}-\nabla_{b} b_{a e c f: d^{f}} \\
& \left.+\nabla_{c} b_{a b d f: e^{f}}-\nabla_{c} b_{a b e f: d^{f}}\right) \\
& E_{l+1}[b]_{a_{1} \cdots a_{l}: b c}=\nabla^{a} b_{a a_{1} \cdots a_{l}: b c}+l^{-1} \nabla^{a}\left(b_{b a_{1} \cdots a_{l}: c a}-b_{c a_{1} \cdots a_{l}: b a}\right) \\
& -\frac{(-1)^{l}}{l}\left(\nabla_{b} b_{a_{1} \cdots a_{l} a: c}{ }^{a}-\nabla_{c} b_{a_{1} \cdots a_{l} a: b^{a}}\right)
\end{aligned}
$$

${ }^{3}$ A differential complex on a manifold $M$ is locally exact if every $x \in M$ has a neighborhood such that the complex restricted to it becomes exact. For example, this condition is fulfilled for the de Rham complex thanks to the Poincaré lemma. 


$$
\begin{gathered}
-\frac{(-1)^{l}}{l(l-1)}\left(\nabla_{\{b\}} b_{\left\{a_{1} \cdots a_{l}\right\} a: c}{ }^{a}-\nabla_{\{c\}} b_{\left\{a_{1} \cdots a_{l}\right\} a: b}{ }^{a}\right) \\
\quad \text { for }(l \geq 2), \text { where } \\
p_{\{b\}} t_{\left\{a_{1} \cdots a_{l}\right\}}=\sum_{i=1}^{l}(-1)^{i+1} p_{a_{i}} t_{a a_{1} \cdots \hat{a}_{i} \cdots a_{l}} \quad\left(\hat{a}_{i} \text { omitted }\right) .
\end{gathered}
$$

Their desired Young symmetry properties are demonstrated in 35. Again, we find the following relations with classical differential operators from relativity. The de Donder operator is $D[h]=E_{1}[h]$. The trace from the Riemann to the Ricci tensors is given by $\bar{R}_{a b}=\bar{R}_{a c: b^{c}}=E_{2}[\bar{R}]_{a b}$. The higher homotopy operators $E_{l}$ do not seem to be part of the classical literature. However, they are essentially modified divergence operators and are thus reminiscent of the de Rham co-differentials.

Finally, the cochain maps $P_{l}=E_{l+1} \circ B_{l+1}+B_{l} \circ E_{l}$ (with the edge cases $P_{0}=E_{1} \circ B_{1}$ and $P_{n}=B_{n} \circ E_{n}$ ) are given by

$$
\begin{aligned}
& P_{0}[v]_{a}=\square v_{a}+k \frac{1}{n} v_{a}, \\
& P_{1}[h]_{a b}=\square h_{a b}-k \frac{2}{n(n-1)} h_{a b}+2 k \frac{g_{a b} \operatorname{tr}[h]}{n(n-1)}, \\
& P_{2}[r]_{a b: c d}=\square r_{a b: c d}-k \frac{2}{n} r_{a b: c d}+2 k \frac{(g \odot \operatorname{tr}[r])_{a b: c d}}{n(n-1)}, \\
& P_{3}[b]_{a b c: d e}=\square b_{a b c: d e}-k \frac{(3 n-7)}{n(n-1)} b_{a b c: d e}-2 k \frac{(g \odot \operatorname{tr}[b])_{a b c: d e}}{n(n-1)}, \\
& P_{4}[b]_{a b c d: e f}=\square b_{a b c d: e f}-k \frac{(4 n-14)}{n(n-1)} b_{a b c d: e f}+2 k \frac{(g \odot \operatorname{tr}[b])_{a b c d: e f}}{n(n-1)}, \\
& P_{l}[b]_{a_{1} \cdots a_{l}: b c}=\square b_{a_{1} \cdots a_{l}: b c}-k \frac{\left(l n-l^{2}+2\right)}{n(n-1)} b_{a_{1} \cdots a_{l}: b c} \\
& +(-)^{l} 2 k \frac{(g \odot \operatorname{tr}[b])_{a_{1} \cdots a_{l}: b c}}{n(n-1)} \quad(l \geq 3) .
\end{aligned}
$$

where we have defined the traces as $\operatorname{tr}[h]=h_{e}{ }^{e}, \operatorname{tr}[r]_{a b}=r_{a e: b}{ }^{e}, \operatorname{tr}[b]_{a b: c}=$ $b_{a b e: c^{e}}{ }, \operatorname{tr}[b]_{a b c: d}=b_{a b c e: d^{e}}$, and $\operatorname{tr}[b]_{a_{1} \cdots a_{l}: b}=b_{a_{1} \cdots a_{l} a: b^{a}}$. The required nullhomotopy identities $P_{l}=E_{l+1} \circ B_{l+1}+B_{l} \circ E_{l}$ (including the edge cases $P_{0}=E_{1} \circ B_{1}$ and $\left.P_{n}=B_{n} \circ E_{n}\right)$ are demonstrated in 35. These identities for $P_{0}[v]$ and $P_{1}[v]$ are well known and are tightly linked with the de Donder gauge fixing condition in linearized gravity [52, 18]. The higher cochain maps and the corresponding identities appear to be new. Though, the identity for $P_{2}[r]$ is related to the non-linear wave equations satisfied by the Riemann and Weyl tensors on any vacuum background, sometimes known as the Lichnerowicz Laplacian [40, Sec.1.3] (see also [14, Sec.7.1], [41, Exr.15.2], 10, Eq.35]).

\subsection{Cohomology with unrestricted and compact supports}

Let us denote the cohomology of the Calabi complex by $H C_{X}^{l}(M, g)$, where $X=$ $c,+,-, f c, p c, s c, t c$ or empty, according to the conventions of Section 2, As in the case of the de Rham complex in Section 3, we will later relate the cohomology with causally restricted supports to that with unrestricted or compact supports. 
It remains still to find a means to calculate these cohomology groups. We will state some results in that direction below, referring to 35 for a fuller discussion.

An important observation is that each of the $P_{l}$ operators is wave-like, that is, it has the same principal symbol as the wave operator $\square_{g}$ with respect to the background Lorentzian metric $g$. This observation has a dual role. First, this means that each of the $P_{l}$ operators is Green hyperbolic [3, 2, while being cochain homotopic to zero, opening the door to using the methods of Section 3 to compute the cohomology with causally restricted supports.

The second role is more subtle:

Remark 1 . Note that the principal symbols of the $B_{l}$ maps in the Calabi complex are actually $\mathrm{GL}(n)$-equivariant and so do not actually involve the background metric $g$. On the other hand, the principal symbols of the cochain maps $P_{l}$ do depend on $g$. This dependence comes purely from the cochain homotopy operators $E_{l}=E_{l}^{g}$ and the identity $P_{l}=P_{l}^{g}=E_{l+1}^{g} \circ B_{l+1}+B_{l} \circ E_{l}^{g}$, where we have used the subscript $g$ to indicate that the background metric was used for covariant differentiation and index raising. On the other hand, we are completely free to define a different set of cochain maps $P_{l}^{g_{R}}=E_{l+1}^{g_{R}} \circ B_{l+1}+B_{l} \circ E_{l}^{g_{R}}$, which now depend on a different metric $g_{R}$ with Riemannian signature. It is crucial to note that the principal symbol of $P_{l}^{g_{R}}$ depends only on the principal symbols of the $E_{l}^{g_{R}}$ and $B_{l}$. So, in fact, it is equal to the principal symbol of $P_{l}^{g}$, but with the Lorentzian metric $g$ replaced by the Riemannian metric $g_{R}$. In other words, each of the $P_{l}^{g_{R}}$ operators is elliptic, since its principal symbol coincides with the Laplace operator $\Delta_{g_{R}}$. Of course, $P_{l}^{g_{R}}$ would differ much more radically from the formulas we have given for $P_{l}^{g}$ in the terms of subleading differential orders.

The ellipticity of the complex (together with a subtler property known as a $\delta$-estimate, discussed in more detail in [51, 35]) results in the following

Proposition 7. Let us denote by $\Gamma\left(C_{l} M\right)$ the space of smooth sections of the tensor bundle $C_{l} M \rightarrow M$. (a) The cohomology $H C^{\bullet}(M, g)$ of the Calabi complex $\left(\Gamma\left(C_{l} M\right), B_{l}\right)$ is isomorphic to the cohomology $H^{\bullet}\left(M, \mathcal{K}_{g}\right)$ of the sheaf $\mathcal{K}_{g}$ of Killing vectors on $(M, g)$. (b) If $(M, g)$ is a simply connected, constant curvature Lorentzian manifold, then $H^{\bullet}\left(M, \mathcal{K}_{g}\right) \cong H^{\bullet}(M) \otimes V_{g}$, where $V_{g}$ is the vector space of all Killing vectors and $H^{\bullet}(M)$ is the de Rham cohomology group.

Killing vectors (or rather covectors in our notation) are solutions $v \in \Gamma\left(T^{*} M\right)$ of the Killing equation $K[v]_{a b}=\nabla_{a} v_{b}+\nabla_{b} v_{a}=0$. On simply connected, constant curvature $n$-dimensional spacetimes, $\operatorname{dim} V_{g}=\left(\begin{array}{c}n+1 \\ 2\end{array}\right)$. Note also that the simple connectedness condition implies that $H^{1}(M)=0$. The precise definition of a sheaf and its cohomology is not of particular importance for the moment. For present purposes, it suffices that the above result, at the very least, answers the question of what $H C^{\bullet}(M, g)$ is for the simply connected versions of Minkowski $\left(\mathbb{R}^{n}\right)$, de Sitter $\left(\mathbb{R} \times S^{n-1}\right.$ for $n \geq 3, \mathbb{R}^{2}$ for $\left.n=2\right)$ and anti-de Sitter $\left(\mathbb{R}^{n}\right)$ spacetimes. The proof, together with a partial discussion of the non-simply connected case, can be found in 35 .

It remains to discuss Calabi cohomology with compact supports $H C_{c}^{\bullet}(M, g)$. First, we note that the chain complex $\left(\Gamma\left(C_{l}^{*} M\right), B_{l}^{*}\right)$ formally adjoint to the Calabi complex has the interesting property that equation $B_{n}^{*}[b]=0$ is equivalent to the $(r a n k-(n-2))$ Killing-Yano equation $Y[w]_{a b c_{4} \cdots c_{n}}=\nabla_{(a} w_{b) c_{4} \cdots c_{n}}$, where 
a solution with $w_{\left[b c_{4} \cdots c_{n}\right]}=w_{b c_{4} \cdots c_{n}}$ is called a $(\operatorname{rank}-(n-2))$ Killing-Yano tensor on $(M, g)$. We define Calabi homology $H_{l}(M, g)$ as the cohomology of this adjoint complex $\left(\Gamma_{c}\left(C_{l}^{*} M\right), B_{l}^{*}\right)$ with compact supports and also locally finite Calabi homology as the cohomology of the adjoint complex $\left(\Gamma\left(C_{l}^{*} M\right), B_{l}^{*}\right)$ with unrestricte supports. Since taking formal adjoints preserves the homotopy identities and ellipticity, appealing to the same arguments as above (again, including a $\delta$-estimate [51, 35]) we also have

Proposition 8. (a) Locally finite Calabi homology $H C_{l}^{l f}(M, g)$ is isomorphic to the cohomology $H^{\bullet}\left(M, \mathcal{K} \mathcal{Y}_{g}\right)$ of the sheaf $\mathcal{K Y}_{g}$ of Killing-Yano tensors on $(M, g)$. (b) If $(M, g)$ is a simply connected, constant curvature Lorentzian manifold, then $H^{\bullet}\left(M, \mathcal{K} \mathcal{Y}_{g}\right) \cong H^{\bullet}(M) \otimes W_{g}$, where $W_{g}$ is the vector space of all Killing-Yano tensors and $H^{\bullet}(M)$ is the de Rham cohomology group.

On simply connected, constant curvature $n$-dimensional spacetimes, $\operatorname{dim} W_{g}=$ $\left(\begin{array}{c}n+1 \\ 2\end{array}\right)$ [49. Furthermore, using Remark 1 and some general results from the theory of elliptic differential complexes (see Example 5.1.11 of [51, which relies on the results of [48]), we have the following generalized Poincaré duality isomorphisms [35:

Proposition 9. When finite dimensional, Calabi homology is the linear dual of Calabi cohomology, $H_{l}(M, g)=H C^{l}(M, g)^{*}$, while Calabi cohomology with compact supports is the linear dual of locally finite Calabi homology, $\mathrm{HC}_{c}^{l}(M, g)=$ $H C_{l}^{l f}(M, g)^{*}$. In both cases, the duality can be exhibited via the non-degeneracy of the pairing descended from the natural pairing between the chains and cochains of corresponding complexes.

\subsection{Cohomology with causally restricted supports}

Recall that, in Section 2, we defined de Rham cohomologies $H_{X}^{p}(M)$ with causally restricted supports $X=+,-, s c, t c, p c$ or $f c$ by restricting the de Rham complex to forms with supports indicated by $X$, with the on-shell cohomologies $H_{\square}^{p}(M)$ and $H_{\square, s c}^{p}(M)$. Substituting the Calabi complex for the de Rham complex and the $P_{l}$ operators for the d'Alembertians $\square$, by direct analogy we can define the causally restricted Calabi cohomologies $H C_{X}^{l}(M, g), H C_{P}^{l}(M, g)$ and $H C_{P . s c}^{l}(M, g)$. We can use the same definitions also in the case of the adjoint Calabi complex, with slightly altered notation. Let the causally restricted Calabi homology $H C_{l}^{X}(M, g)$ be the cohomology of the complex $\left(\Gamma_{Y}\left(C_{l}^{*} M\right), B_{l}^{*}\right)$ where the pair $(X, Y)$ is one of retarded $(+, f c)$, advanced $(-, p c)$, spacelike locally finite $(s l f, t c)$, timelike locally finite $(t l f, s c)$, future locally finite $(f l f,-)$ and past locally finite $(p l f,+)$. Similarly, we define the on-shell Calabi homologies $H C_{l}^{P, l f}(M, g)$ and $H C^{P, t l f}(M, g)$ as the cohomologies of the complexes $\left(\operatorname{ker} P_{l}^{*} \cap \Gamma\left(C_{l}^{*} M\right), B_{l}^{*}\right)$ and $\left(\operatorname{ker} P_{l}^{*} \cap \Gamma_{s c}\left(C_{l}^{*} M\right), B_{l}^{*}\right)$, respectively. The above $(X, Y)$ pairs are chosen specifically so that there is a bilinear pairing between Calabi homology $H_{l}^{X}(M, g)$ and Calabi cohomology $H C_{Y}^{l}(M, g)$, which descends from the natural pairing between the corresponding spaces of sections of $C_{l}^{*} M$ and $C_{l} M$.

The specific way in which these causally restricted cohomologies are of importance in linearized gravity is summarized in the following proposition. For definiteness of notation let us fix a $\chi \in C^{\infty}(M)$ that is 1 in the future of a 
Cauchy surface $\Sigma_{+}$and 0 in the past of another Cauchy surface $\Sigma_{-}$. The following is a special case of the general result [36, Thm.3.2].

Proposition 10. Linearized gravity on a constant curvature background 36, Sec.4.4] induces a symplectic form on $\Gamma_{P, s c}\left(C_{1} M\right)$ [36, Def.3.10] that is nondegenerate when (a) the bilinear form on $H C_{s c}^{1}(M, g) \times H C_{1}(M, g)$ induced by $\langle\alpha, \beta\rangle=\int_{M} \alpha \cdot \beta$ is non-degenerate and (b) the bilinear form on $H C_{P, s c}^{1}(M)$ induced by $\langle\alpha, \beta\rangle_{P}=\int_{M} \alpha \cdot P_{1}[\chi \beta]$ is non-degenerate.

From the proof of that proposition it also follows that degeneracies in (a) and (b) can imply degeneracies in the corresponding (pre-)symplectic structure.

With the above discussion in mind, we can see immediately that we are in a situation very similar to that of Section 3, with the de Rham complex replaced by the Calabi complex (or its adjoint complex) and the wave operators $\square$ replaced by the operators $P_{l}$ (or $P_{l}^{*}$ ), which have wave-like principal symbols and are Green hyperbolic. So, repeating the arguments of Section 3. we immediately have the following

Theorem 11. Consider a globally hyperbolic, constant curvature Lorentzian manifold $(M, g)$. The Calabi cohomology $H C_{X}^{l}(M, g)$ with the causally restricted supports $X=+,-, p c$ or $f c$ is trivial. Moreover, for the cases $X=s c, t c$, we have the isomorphisms

$$
\begin{aligned}
H C_{s c}^{l}(M, g) \cong H C_{c}^{l+1}(M, g), \quad H C_{P, s c}^{l}(M, g) \cong H C_{c}^{l}(M, g) \oplus H C_{c}^{l+1}(M, g), \\
H C_{t c}^{l}(M, g) \cong H C^{l-1}(M, g), \quad H C_{P}^{l}(M, g) \cong H C^{l}(M, g) \oplus H C^{l-1}(M, g),
\end{aligned}
$$

with the convention that all cohomologies vanish in degree $l$ for $l<0$ or $l>n$. Similarly, the Calabi homology $\mathrm{HC}_{l}^{X}(M, g)$ with the causally restricted supports $X=+,-$,plf or flf is trivial. Moreover, for the cases $X=t l f$, slf, we have the isomorphisms

$$
\begin{aligned}
& H C_{l}^{t l f}(M, g) \cong H C_{l-1}(M, g), H C_{P, t l f}^{l}(M, g) \cong H C_{l}(M, g) \oplus H C_{l-1}(M, g), \\
& H C_{l}^{s l f}(M, g) \cong H C_{l+1}^{l f}(M, g), \quad H C_{P, l f}^{l}(M, g) \cong H C_{l}^{l f}(M, g) \oplus H C_{l+1}^{l f}(M, g),
\end{aligned}
$$

again with the convention that all cohomologies vanish in degree $l$ for $l<0$ or $l>n$.

The Calabi cohomology with spacelike compact support in degree $l=1$ is important in understanding the symplectic and Poisson structure of the classical field theory (and of course the quantization) of linearized gravitons on a background of constant curvature. This was pointed out explicitly in [36, Sec.4.4] as a special case of a more general phenomenon (also discussed in [34).

Remark 2. Using the above theorem and the results of Section 4.3, we can assert that for $n$-dimensional Minkowski space $H C_{s c}^{l}$ vanishes in all degrees except $l=n-1$, while $H C_{P, s c}^{l}$ vanishes in all degrees except $l=n, n-1$. For $n$-dimensional de Sitter space $H C_{s c}^{l}$ vanishes in all degrees except $l=n-1$, while $H C_{P, s c}^{l}$ vanish in all degrees except $l=0, n-1, n$. Similar remarks apply to Calabi homologies. 


\section{$5 \quad$ Notes and generalizations}

\subsection{Generalized causal structures}

The notion of a causal structure on a manifold or even a topological space (in the sense of a partial order on events) can be generalized quite fare beyond the context of Lorentzian geometry 37,22 . We will stick with the context of differential geometry, where a natural generalization consists of introducing at every point of a manifold an arbitrary convex cone in the tangent 4 bundle. Such a manifold could be called a conal manifold [42, 38, 50, 34]. Various notions generated by the causal structure on Lorentzian manifolds survive almost without modification on conal manifolds, including spacelike and timelike compactness. The main question we will try to answer in this section is the following: Is it possible to use the methods of Section 3 to compute causally restricted cohomologies on a conal manifold? We shall see that the answer is yes, even if the conal manifold is not Lorentzian.

\subsubsection{Conal manifolds}

Before dealing with spacelike and timelike compactly supported forms, let us introduce the basics of conal manifolds and causal relations on them. Let $M$ be a smooth manifold and $C \subset T M$ be an open subset, such that $C_{x}=C \cap T_{x} M$ is an open, convex cone in $T_{x} M$ that does not contain any affine line. It can be shown that the interior $C_{x}^{\circledast}$ of the polar dual (or convex dual) cone $T_{x}^{*} M \supset C_{x}^{*}=\left\{p \in T_{x}^{*} M \mid \forall v \in C_{x}: p \cdot v \geq 0\right\}$ satisfies the same conditions, with $C^{\circledast}=\sqcup_{x \in M} C_{x}^{\circledast}$. The pair $(M, C)$ or $\left(M, C^{\circledast}\right)$ is called a conal manifold, with $C$ (or $C^{\circledast}$ ) called the tangent (or cotangent) cone distribution or cone bundle. For example, the subset of non-vanishing, future-pointing, timelike vectors on a Lorentzian manifold with a time orientation satisfies the above conditions. In general, the cones $C_{x}$ need not even have elliptic cross sections, thus not be associated to any Lorentzian metric. The cones of future pointing timelike vectors of linear symmetric hyperbolic PDE systems also satisfy the same properties [34, Sec.4.1]. Sometimes, it is also convenient to admit degenerate cases where the cones are not open or contain some affine lines, but some special care must be taken in those situations.

Given a conal manifold $(M, C)$ we can define a chronological order relation on the points of $M$. Namely, $x \ll y$ if there exists a smooth curve $\gamma:[0,1] \rightarrow M$, such that $\gamma(0)=x, \gamma(1)=y$ and $\dot{\gamma}(t) \in C$ for all $t \in[0,1]$. It can be shown that the chronological order relation $I^{+} \subset M \times M$ is open and transitive. We can also define the reverse chronological order, $I^{-}$, and chronological influence, $I=$ $I^{+} \cup I^{-}$, relations in the obvious way. We avoid defining the analog of the causal order relation usually denoted by $J^{+}$, simply because we have not made any hypotheses about the regularity of the set of causal vectors $\left(\bar{C}_{x} \subset T_{x} M\right)$. Given any set $K \subseteq M$, we denote by $I^{ \pm}(K)$ the set of all points of $M$ that respectively chronologically precede ore are preceded by the points of $K$. In general, $I^{ \pm}(K)$ is not closed, even if $K$ is. So, for convenience we define $\bar{I}^{ \pm}(K)=\overline{I^{ \pm}(K)}$. We also use the notation $I(K)=I^{+}(K) \cup I^{-}(K)$ and $\bar{I}(K)=\bar{I}^{+}(K) \cup \bar{I}^{-}(K)$. Note that $\bar{I}^{ \pm} \subseteq M \times M$ need not be transitive as relations.

\footnotetext{
${ }^{4}$ One could equally do so in the cotangent bundle, and produce a tangent cone by convex (or polar) duality.
} 
The definition of a Cauchy surface $\Sigma \subset M$ is the usual one, every inextensible smooth curve with timelike tangents must intersect $\Sigma$ exactly once. It has recently been shown that the smooth version of the Geroch splitting theorem 25 , 9, 8] generalizes to conal manifolds [17]. So, globally hyperbolicity can be simply characterized by the existence of a Cauchy surface. Also, the results of [46] should also directly carry over to conal manifolds. Finally, we define the notions of advanced, retarded, spacelike compact, timelike compact, future compact and past compact exactly in the same way as in Section 2, with the exception that we use the relations $\bar{I}^{ \pm}$and $\bar{I}$ instead of the relations $J^{ \pm}$and $J$

\subsubsection{Cohomology with causally restricted supports}

Let $M$ be a globally hyperbolic conal manifold and $g$ an auxiliary globally hyperbolic Lorentzian metric that induces another conal structure on $M$ that is "slower" than the original one. That is, $\Omega_{ \pm_{g}}^{p}(M) \subseteq \Omega_{ \pm}^{p}(M)$, which also implies that $\Omega_{s c_{g}}^{p}(M) \subseteq \Omega_{s c}^{p}(M)$, while $\Omega_{f c_{g}, p c_{g}}^{p}(M) \supseteq \Omega_{f c, p c}^{p}(M)$, and hence $\Omega_{t c_{g}}^{p}(M) \supseteq$ $\Omega_{t c}^{p}(M)$. Any conal manifold admits a nowhere vanishing vector field (contract each cone to a ray and select a vector from it), which is moreover everywhere future directed. So, the existence of such an auxiliary Lorentzian metric follows from the same known, general arguments that show the existence of Lorentzian metrics on manifolds of vanishing Euler characteristic (i.e., admitting a nowhere vanishing vector field) [4, 43. The "slowness" requirement is implemented by making sure that the Lorentzian timelike cones closely hug the directions singled out by the above everywhere timelike vector field.

Let $\mathrm{G}_{ \pm}$denote once again the advanced and retarded Green functions of the wave operator $\square_{g}$ defined with respect to $g$. Then it is easy to see that the Green functions are still well defined and injective as maps $\mathrm{G}_{ \pm}: \Omega_{c}^{p}(M) \rightarrow$ $\Omega_{ \pm}^{p}(M)$. Appealing to the same logic as in the standard proof $6[3,26,36,34,2$, we can extend the Green functions to bijective maps $\mathrm{G}_{ \pm}: \Omega_{ \pm}^{p}(M) \rightarrow \Omega_{ \pm}^{p}(M)$ and $\mathrm{G}_{ \pm}: \Omega_{f c, p c}^{p}(M) \rightarrow \Omega_{f c, p c}^{p}(M)$, from which it is straightforward to establish exactness of the following sequences, with $\mathrm{G}=\mathrm{G}_{+}-\mathrm{G}_{-}$:

$$
\begin{aligned}
& 0 \longrightarrow \Omega_{0}^{p}(M) \stackrel{\square}{\longrightarrow} \Omega_{0}^{p}(M) \stackrel{\mathrm{G}}{\longrightarrow} \Omega_{s c}^{p}(M) \stackrel{\square}{\longrightarrow} \Omega_{s c}^{p}(M) \longrightarrow 0, \\
& 0 \longrightarrow \Omega_{t c}^{p}(M) \stackrel{\square}{\longrightarrow} \Omega_{t c}^{p}(M) \stackrel{\mathrm{G}}{\longrightarrow} \Omega^{p}(M) \stackrel{\square}{\longrightarrow} \Omega^{p}(M) \longrightarrow 0,
\end{aligned}
$$

where the supports are restricted by the given conal structure on $M$ and not by that induced by the auxiliary Lorentzian metric $g$. Note that the proofs would make use of the hypothesis that the given conal structure is globally hyperbolic, specifically in the construction of explicit splitting maps that demonstrate exactness [36, Lem.2.1]. Thus, repeating the arguments Section 3, we establish the following generalization of Theorems 2 and 3 .

\footnotetext{
${ }^{5}$ We are not concerned with possible minor inconsistencies this substitution introduces in the case of Lorentzian manifolds with ill-behaved causal structures. In any case, we shall only apply these notions for globally hyperbolic spacetimes, where these differences do not appear.

${ }^{6}$ Pick an exaustion of $M$ by compact sets and adapt a sequence of smooth "step functions" to this exaustion. Precomposing $G_{ \pm}$with multiplication by these step functions gives a sequence of operators which converges to an operator with the desired extended domain.
} 
Theorem 12. Consider a globally hyperbolic conal manifold $M$. Its de Rham cohomology $H_{X}^{p}(M)$ with causally restricted supports $X=+,-, p c$ or $f c$ is trivial. Moreover, we have the isomorphisms

$$
\begin{aligned}
H_{s c}^{p}(M) & \cong H_{c}^{p+1}(M), & H_{\square, s c}^{p} & \cong H_{c}^{p}(M) \oplus H_{c}^{p+1}(M), \\
H_{t c}^{p}(M) & \cong H^{p-1}(M), & \text { and } H_{\square}^{p}(M) & \cong H^{p}(M) \oplus H^{p-1}(M),
\end{aligned}
$$

with the convention that all cohomologies vanish in degree $p$ for $p<0$ or $p>n$.

It should be clear from the preceding discussion that there is nothing inherently special in our use of the d'Alembertian $\square_{g}$, when it comes to the calculation of de Rham cohomologies with causally restricted supports on a globally hyperbolic conal manifold $M$. It is merely one of multiple possible auxiliary hyperbolic differential operators that can serve the same purpose. Here are the key required properties for such an operator $h$ : (a) $h$ must be a cochain map that is homotopic to zero with respect to the de Rham complex, (b) it must possess retarded and advanced Green functions, (c) these Green functions must be causal with respect to the given conal structure on $M$. In fact, the conclusion of our Theorem 3 was reached independently in the recent paper [5] by following an argument structurally similar to ours, with the d'Alembertian replaced by the Lie derivative $\mathcal{L}_{v}$ with respect to a complete timelike vector field $v$. It is clearly (Green) hyperbolic [3, 2, 34, 36] with Green functions simply given by integration (into the future or past) along the flow lines of $v$. Moreover, it is cochain homotopic to zero because of the well known magic formula of Cartan: $\mathcal{L}_{v}=\iota_{v} \mathrm{~d}+\mathrm{d} \iota_{v}$.

\subsection{Functoriality}

Recall that ordinary de Rham cohomology is defined on any finite dimensional manifold and the pullback of differential forms along a smooth map between manifolds induces a map between their cohomologies (in the direction opposite the original smooth map). This observation has the following well-known formalisation: de Rham cohomology in degree $p, H^{p}(-)$, is a contravariant functor 7 from the category of smooth manifolds to the category of real vector spaces. The same cannot be said for de Rham cohomology with compact supports, $H_{c}^{p}(-)$, because the pullback of a compactly supported form need not be compactly supported itself. This pullback problem is fixed by considering only proper 8 smooth maps between manifolds. So, given a proper smooth map $f: M \rightarrow N$, pullback along it induces a contravariant map between de Rham cohomologies in degree $p$ with compact support, $f^{*}: H_{c}^{p}(N) \rightarrow H_{c}^{p}(M)$. If the map $f$ satisfies a different restrictive condition, namely that it is an open embedding, it is possible to define a covariant pushforward map $f_{*}: H_{c}^{p}(M) \rightarrow H_{c}^{p}(N)$ : we can identify $M$ with its image $f(M)$, an open subset of $N$, and extend by zero any compactly supported form defined $M$ to all of $N$. In short, de Rham cohomology with compact supports, $H_{c}^{p}(-)$, defines a contravariant functor on the category of

\footnotetext{
${ }^{7} \mathrm{We}$ shall not delve here into the details of category theory. It suffices to say that any statement that we shall make involving functors and categories will be simply a very terse transcription of some other property that will be spelled out in more elementary terms. More details about the functorial properties of de Rham cohomology can be found in [1].

${ }^{8} \mathrm{~A}$ continuous map is proper if the preimage of any compact set is compact.
} 
smooth manifolds with proper maps as morphisms, when paired with the pullback, while it defines a covariant functor on the category of smooth manifolds with open embeddings as morphisms, when paired with the pushforward.

A natural question is the following: do similar properties hold, and under what precise conditions, for de Rham cohomologies with causally restricted supports? For instance, this question was briefly raised, but without any definite answer, in [5]. In fact, it is straight forward to present causally restricted cohomologies as functors, provided we modify the domain category by adding generalized causal structures to manifolds (as in Section 5.1) and by modifying the notion of a proper map with respect to the causal structure.

Consider two conal manifolds $M$ and $N$, with a smooth map $f: M \rightarrow N$ between them. We call the map $f$ reflectively spacelike-proper if the preimage of any spacelike compact set is also spacelike compact, while we call it reflectively timelike-proper if the preimage of any timelike compact set is also timelike compact. When the map $f$ is an open embedding, we also introduce the terminology monotonically spacelike-proper for the case when the image of any spacelike compact set is itself spacelike-compact and monotonically timelike-proper for the case when the image of any timelike compact set is timelike compact. We should note that the above terminology is partly inspired by some general notions from the theory of partially ordered sets. A map $f: M \rightarrow N$ between two partially ordered sets $(M, \leq)$ and $(N, \leq)$ is said to be monotonic if $x \leq y$ implies $f(x) \leq f(y)$ and, on the other hand, it is said to be order-reflecting if $f(x) \leq f(y)$ implies $x \leq y$. The following theorem is a straight forward generalization of the previous arguments for the simpler case of compact supports.

Theorem 13. Let $\mathfrak{C M a n}_{s c}$ and $\mathfrak{C M a n}_{t c}$ be the categories of conal manifolds with, respectively, reflectively spacelike-proper and reflectively timelike-proper, smooth maps as morphisms, while the $\mathfrak{C M a n}_{s c}^{e}$ and $\mathfrak{C M a n}_{t c}^{e}$ categories have, respectively, monotonically spacelike-proper and monotonically timelike-proper open embeddings as morphisms. Then, de Rham cohomologies with spacelike and timelike supports, $H_{s c}^{p}(-)$ and $H_{t c}^{p}(-)$, are contravariant functors on $\mathfrak{C M a n}_{s c}$ and $\mathfrak{C M a n}_{t c}$, respectively. Similarly, $H_{t c}^{p}(-)$ and $H_{s c}^{p}(-)$ are covariant functors on $\mathfrak{C M a n}_{t c}^{e}$ and $\mathfrak{C M a n}_{s c}^{e}$, respectively.

Proof. The proof is a direct parallel of the above arguments for the case with compact supports, since the definitions have been specifically adapted to that argument.

To show that the definitions of spacelike- and timelike-proper maps are in some sense natural, we give a couple of examples.

Lemma 14. Let $M$ be a manifold and two conal structures on it, $C \subseteq C^{\prime} \subseteq$ $T M$ ( $C$ is "slower" than $C^{\prime}$ ) (Section 5.1). The identity map is a reflectively spacelike-proper from $\left(M, C^{\prime}\right)$ to $(M, C)$ and reflectively timelike-proper from $(M, C)$ to $\left(M, C^{\prime}\right)$

Proof. Let $K \subseteq M$ be any compact subset. Then, by hypothesis, the $C$-influence set is smaller than the $C^{\prime}$-influence set, $\bar{I}_{C}(K) \subseteq \bar{I}_{C^{\prime}}(K)$. Therefore, any $C$ spacelike compact set is also $C^{\prime}$-spacelike and hence the identity from $\left(M, C^{\prime}\right)$ to $(M, C)$ is reflectively spacelike-proper. On the other hand, if $U \subseteq M$ is $C^{\prime}$ timelike compact, then we have the inclusion $\bar{I}_{C}(K) \cap U \subseteq \bar{I}_{C^{\prime}}(K) \cap U$, the 
latter being compact. Therefore, $U$ is also $C$-timelike compact and the identity from $(M, C)$ to $\left(M, C^{\prime}\right)$ is reflectively timelike-proper.

Lemma 15. Let $(M, g)$ and $(N, h)$ be two globally hyperbolic Lorentzian manifolds and $f: M \rightarrow N$ an open isometric embedding, such that the image of a Cauchy surface of $M$ is a Cauchy surface of $N$. Then, $f$ is monotonically timelike-proper.

Proof. Let $U \subseteq M$ be timelike compact. According to 46 , this is equivalent to $U$ being contained between two Cauchy surfaces in $(M, g)$, say $\Sigma_{1}, \Sigma_{2} \subset M$. This means that the image, $f(U)$ is contained between $f\left(\Sigma_{1}\right)$ and $f\left(\Sigma_{2}\right)$, with the latter, by hypothesis, being Cuachy surfaces in $(N, h)$. Thus, $f(U)$ is also timelike compact and the map $f$ is monotonically timelike-proper.

\subsection{Other differential complexes}

Our interest in computing the de Rham and Calabi cohomologies with causally restricted supports has was motivated by their importance in understanding the geometric structure of classical and quantum field theories [15, 47, 6, 19, 5, 31, 34, 36. Namely, for a general class of linear field theories, one can formulate sufficient conditions for the non-degeneracy of the theory's Poisson structure and the completeness of compactly supported smeared fields as physical observables in terms of the cohomologies of corresponding differential complexes. Non-linear field theories can be studied in terms of their linearizations about arbitrary background solutions. To Maxwell electrodynamics corresponds the de Rham complex [36, Sec.4.2]. To linearized gravity on constant curvature backgrounds, corresponds the Calabi complex [36, Sec.4.4]. Similarly, to Yang-Mills linearized about a flat connection corresponds a twisted de Rham complex.

Each of these examples can be treated using the methods presented in this paper. Few other explicit examples of differential complexes corresponding to other field theories of physical interest seem to be known. In particular, they do not seem to be known for linearized gravity on non-constant curvature backgrounds and, perhaps, not even for Yang-Mills linearized about non-flat connections. On the other hand, there are strong abstract reasons to believe that such differential complexes do indeed exist 44, 28, 44 .

If such a differential complex also shares the apparently crucial property of admitting cochain homotopies that generate hyperbolic and elliptic cochain maps (cf. the $E_{l}^{g}, P_{l}^{g}, E_{l}^{g_{R}}$ and $P_{l}^{g_{R}}$ maps of Sections 4.2 and 4.3), then its causally restricted cohomologies can be related to those with unrestricted and compactly supported ones, as in Theorems 3 and 11 .

If, in addition, such a differential complex could also be seen as resolving a locally constant sheaf, its unrestricted cohomologies could be computed by algebraic means, without actually solving complicated systems of differential equations, as in Section 4.3. The latter requirement is closely related to the initial differential operator in the complex having only a finite dimensional space of solutions (being of finite type), as is the case for the locally constant (de Rham) and Killing (Calabi) conditions.

The compactly supported cohomologies could also be obtained if the corresponding formally adjoint complex satisfied similar requirements, as illustrated in Section 4.3 by the appearance of the locally constant sheaf of Killing-Yano tensors. 


\section{Discussion}

We have shown how to compute the de Rham cohomology with causally restricted supports (retarded, advanced, past compact, future compact, spacelike compact and timelike compact) on a globally hyperbolic Lorentzian spacetime, using special properties of the d'Alembert wave operator and its Green functions. The result (Theorems 2, 3 and Corollary 5) expresses these causally restricted cohomologies in terms of the standard de Rham cohomologies of the spacetime manifold, with either unrestricted or compact supports. These results, confirm the independent similar results of the recent work 5. However, since our method does not rely on the strong invariance properties of the de Rham complex under topological homotopies, we have also obtained further results. In particular, our method is also applicable to the Calabi complex (Theorem 11). The Calabi complex appears in linearized gravity on constant curvature backgrounds in a way similar to the de Rham complex in Maxwell theory. These results answer some questions that have naturally arisen in recent investigations of classical and quantum gauge theories on curved spacetimes.

Finally, we have also made comments about other questions that have naturally appeared in these investigations. Namely, we discussed the covariance of causally restricted cohomologies under specific types of morphisms between spacetimes, adapted to their causal structure, and under changes of the causal structure itself.

We have presented almost the bare minimum of information about the Calabi complex that is needed to obtain our results. A fuller discussion of this interesting complex, including relevant geometric properties that are difficult to locate in or are absent from the current literature, is deferred to future work [35. In the future, it will also be interesting to find the analogs of the Calabi complex on more general Lorentzian backgrounds, which would consist of differential complexes resolving the sheaf of Killing vectors on a given background. However, we conjecture that the Hodge-like structure that we have used to compute causally restricted cohomologies will be shared by all of them.

\section{Acknowledgments}

The author would like to thank Marco Benini for useful discussions and also Romeo Brunetti, Claudio Dappiaggi, and Valter Moretti for their support in the course of this work.

\section{References}

[1] A. Ashtekar and A. Sen, "On the role of spacetime topology in quantum phenomena: Superselection of charge and emergence of nontrivial vacua," Journal of Mathematical Physics 21 (2008) 526-533.

[2] C. Baer, "Green-hyperbolic operators on globally hyperbolic spacetimes." 2013. arXiv:1310.0738

[3] C. Baer, N. Ginoux, and F. Pfaeffle, Wave Equations on Lorentzian Manifolds and Quantization, vol. 2 of ESI 
lectures in mathematics and physics. European Mathematical Society, 2007. arXiv:0806.1036.

[4] J. K. Beem, P. Ehrlich, and K. Easley, Global Lorentzian Geometry, vol. 202 of Pure and Applied Mathematics. Marcel Dekker, New York, 1996.

[5] M. Benini, "Optimal space of linear classical observables for Maxwell $k$-forms via spacelike and timelike compact de Rham cohomologies." 2014. arXiv:1401.7563

[6] M. Benini, C. Dappiaggi, and A. Schenkel, "Quantized Abelian principal connections on Lorentzian manifolds," Communications in Mathematical Physics 330 (2013) 123-152, arXiv:1303.2515.

[7] L. B. Bergery, J. P. Bourguignon, and J. Lafontaine, "Déformations localement triviales des variétés riemanniennes," in Differential Geometry, Part 1, vol. 27 of Proceedings of Symposia in Pure Mathematics, pp. 3-32. AMS, Providence, RI, 1975.

[8] A. Bernal and M. Sánchez, "Further results on the smoothability of cauchy hypersurfaces and cauchy time functions," Letters in Mathematical Physics 77 (2006) 183-197, arXiv:gr-qc/0512095.

[9] A. N. Bernal and M. Sánchez, "Smoothness of time functions and the metric splitting of globally hyperbolic spacetimes," Communications in Mathematical Physics 257 (2005) 43-50 arXiv: gr-qc/0401112.

[10] D. Bini, C. Cherubini, R. T. Jantzen, and R. Ruffini, "de Rham wave equation for tensor valued $p$-forms," International Journal of Modern Physics D 12 (2003) 1363-1384.

[11] R. Bott and L. W. Tu, Differential Forms in Algebraic Topology, vol. 82 of Graduate Texts in Mathematics. Springer, New York, 1982.

[12] G. E. Bredon, Sheaf Theory. Graduate Texts in Mathematics. Springer, New York, 1997.

[13] E. Calabi,

"On compact, Riemannian manifolds with constant curvature. I," in Differential Geometry, C. B. Allendoerfer, ed., vol. 3 of Proceedings of Symposia in Pure Mathematics, pp. 155-180. AMS, Providence, RI, 1961.

[14] D. Christodoulou and S. Klainerman, The global nonlinear stability of the Minkowski space, vol. 41 of Princeton Mathematical Series. Princeton University Press, Princeton, NJ, 1993.

[15] C. Dappiaggi and B. Lang, "Quantization of Maxwell's equations on curved backgrounds and general local covariance," Letters in Mathematical Physics 101 (2012) 265-287, arXiv:1104.1374. 
[16] M. Eastwood, "Variations on the de Rham complex," Notices of the American Mathematical Society 46 (1999) 1368-1376. http://www .ams.org/notices/199911/fea-eastwood.pdf.

[17] A. Fathi and A. Siconolfi, "On smooth time functions," Mathematical Proceedings of the Cambridge Philosophical Society 152 (2011) 303-339

[18] C. J. Fewster and D. S. Hunt, "Quantization of linearized gravity in cosmological vacuum spacetimes," Reviews in Mathematical Physics 25 (2013) 1330003, arXiv:1203.0261.

[19] C. J. Fewster and B. Lang, "Dynamical locality of the free Maxwell field." 2014. arXiv: 1403.7083

[20] S. A. Fulling, R. C. King, B. G. Wybourne, and C. J. Cummins, "Normal forms for tensor polynomials. I. The Riemann tensor," Classical and Quantum Gravity 9 (1992) 1151

[21] W. Fulton, Young Tableaux: With Applications to Representation Theory and Geometry, vol. 35 of London Mathematical Society Student Texts. Cambridge University Press, New York, 1997.

[22] A. García-Parrado and J. M. M. Senovilla, "Causal structures and causal boundaries," Classical and Quantum Gravity 22 (2005) R1-R84, arXiv:gr-qc/0501069

[23] J. Gasqui and H. Goldschmidt, "Déformations infinitésimales des espaces riemanniens localement symétriques. I," Advances in Mathematics 48 (1983) 205-285.

[24] J. Gasqui and H. Goldschmidt, "Complexes of differential operators and symmetric spaces," in Deformation Theory of Algebras and Structures and Applications, M. Hazewinkel and M. Gerstenhaber, eds., vol. 247 of NATO ASI Series, pp. 797-827. Kluwer, Dordrecht, 1988.

[25] R. Geroch, "Domain of dependence," Journal of Mathematical Physics 11 (1970) 437-449

[26] N. Ginoux, "Linear wave equations," in Quantum Field Theory on Curved Spacetimes: Concepts and Methods, C. Baer and K. Fredenhagen, eds., vol. 786 of Lecture Notes in Physics. Springer, 2009.

[27] S. I. Goldberg, Curvature and homology. Dover, Mineola, NY, 1998.

[28] H. Goldschmidt, "Existence theorems for analytic linear partial differential equations," The Annals of Mathematics 86 (1967) 246-270.

[29] H. Goldschmidt, "Duality theorems in deformation theory," Transactions of the American Mathematical Society 292 (1985) 1.

[30] T.-P. Hack, "Quantization of the linearised Einstein-Klein-Gordon system on arbitrary backgrounds and the special case of perturbations in inflation." 2014. arXiv:1403.3957. 
[31] T.-P. Hack and A. Schenkel, "Linear bosonic and fermionic quantum gauge theories on curved spacetimes," General Relativity and Gravitation 45 (2013) 877-910, arXiv:1205.3484

[32] S. W. Hawking and G. F. R. Ellis, The Large Scale Structure of Space-Time. Cambridge Monographs on Mathematical Physics. Cambridge University Press, Cambridge, London, 1973.

[33] J. Jost, Riemannian Geometry and Geometric Analysis. Springer, Berlin, 2011.

[34] I. Khavkine, "Characteristics, conal geometry and causality in locally covariant field theory." 2012. arXiv:1211.1914

[35] I. Khavkine, "The Calabi complex and Killing sheaf cohomology." 2014. arXiv:1409.7212

[36] I. Khavkine, "Covariant phase space, constraints, gauge and the Peierls formula," International Journal of Modern Physics A 29 (2014) 1430009, arXiv: 1402.1282

[37] E. H. Kronheimer and R. Penrose, "On the structure of causal spaces," Mathematical Proceedings of the Cambridge Philosophical Society 63 (1967) 481-501.

[38] J. D. Lawson, "Ordered manifolds, invariant cone fields, and semigroups," Forum Mathematicum 1 (1989) 273-308.

[39] P. Leyland and J. E. Roberts, "The cohomology of nets over Minkowski space," Communications in Mathematical Physics 62 (1978) 173-189.

[40] A. Lichnerowicz, "Propagateurs, commutateurs et anticommutateurs en relativité générale," in Relativity, Groups and Topology, C. DeWitt and B. S. DeWitt, eds., pp. 821-861. Gordon and Breach, New York, 1964.

[41] C. W. Misner, K. S. Thorne, and J. A. Wheeler, Gravitation. Physics Series. W. H. Freeman, San Francisco, 1973.

[42] K. H. Neeb, "Conal orders on homogeneous spaces," Inventiones Mathematicae 104 (1991) 467-496.

[43] B. O'Neill, Semi-Riemannian Geometry With Applications to Relativity, vol. 103 of Pure and Applied Mathematics. Academic Press, San Diego, 1983.

[44] J.-F. Pommaret, Systems of partial differential equations and Lie pseudogroups, vol. 14 of Mathematics and Its Applications. Gordon and Breach, New York, 1978.

[45] D. G. Quillen, Formal properties of over-determined systems of partial differential equations. PhD thesis, Harvard University, 1964.

[46] K. Sanders, "A note on spacelike and timelike compactness," Classical and Quantum Gravity 30 (2012) 115014, arXiv:1211.2469 
[47] K. Sanders, C. Dappiaggi, and T.-P. Hack, "Electromagnetism, local covariance, the Aharonov-Bohm effect and Gauss' law,"

Communications in Mathematical Physics 328 (2014) 625-667, arXiv:1211.6420

[48] J.-P. Serre, "Un théorème de dualité,"

Commentarii Mathematici Helvetici 29 (1955) 9-26.

[49] S. E. Stepanov, "The Killing-Yano tensor,"

Theoretical and Mathematical Physics 134 (2003) 333-338.

[50] D. Sullivan, "Cycles for the dynamical study of foliated manifolds and complex manifolds," Inventiones Mathematicae 36 (1976) 225-255.

[51] N. N. Tarkhanov, Complexes of Differential Operators, vol. 340 of Mathematics and Its Applications. Kluwer, Dordrecht, 1995.

[52] R. M. Wald, General Relativity. University of Chicago Press, Chicago, 1984. 